\title{
TikTok: usos e motivações entre adolescentes em Portugal
}

TikTok: usos y motivaciones entre los adolescentes en Portugal

TikTok: uses and motivations among teenagers in Portugal

\author{
Alexandre DUARTE \\ Centro de Estudos Sociedade e Comunicação - Portugal \\ alexandre_arte@yahoo.co.uk \\ Patrícia DIAS \\ CECC - Universidade Católica Portuguesa - Portugal \\ pdias@fch.lisboa.ucp.pt
}

Chasqui. Revista Latinoamericana de Comunicación

$N .^{o}$ 147, agosto-noviembre 2021 (Sección Monográfico, pp. 81-102)

ISSN 1390-1079 / e-ISSN 1390-924X

Ecuador: CIESPAL

Recibido: 09-02-2021 / Aprobado: 10-07-2021 


\title{
Resumo
}

Durante os primeiros meses de confinamento devido à pandemia Covid-19, o TikTok foi a aplicação mais descarregada a nível mundial, tendo conquistado, só em Portugal, meio milhão de novos utilizadores, o que requer um olhar mais aprofundado por parte das Ciências de Comunicação. Usando como base a teoria dos Usos e Gratificações (U\&G), este estudo pretende mapear as práticas de adolescentes portugueses (13-16) nesta plataforma, explorando as diferenças entre os utilizadores e os criadores de conteúdos. Para tal, foi realizada um inquérito online a uma amostra não probabilística de 263 utilizadores do TikTok. Os resultados mostram que $44,5 \%$ produzem conteúdos e que estes têm uma atuação mais estratégica, focada na autopromoção, na expansão e dinamização da sua comunidade online.

Palavras-chave: TikTok, adolescentes (13-16), aplicação móvel, conteúdos gerados pelos utilizadores (UGC), media gerados pelo utilizador (UGM), Usos e Gratificações (U\&G).

\begin{abstract}
During the first months of lockdown due to the Covid-19 pandemic, TikTok was the most downloaded app worldwide, and conquered, just in Portugal, half a million new users, which requires an attentive look from Media Studies. Using the Uses and Gratifications (U\&G) as framework, this study aims to map the practices of Portuguese teenagers (13-16) on this platform, exploring the differences between users and content producers. For that purpose, we conducted an online survey to a non-probabilistic sample of 263 TikTok users. Our findings show that $44,5 \%$ of the users create content, and they act more strategically, focusing on self-promotion and on the expansion and dynamization of their online community.

Keywords: TikTok, teenagers (13-16), mobile apps, user-generated content (UGC), media-generated content (UGM), Uses and Gratifications (U\&G);

\section{Resumen}

Durante el confinamiento por la pandemia Covid-19, TikTok fue la aplicación más descargada en todo el mundo, habiendo conquistado, solo en Portugal, medio millón de nuevos usuarios, lo que requiere una mirada más profunda por parte de las ciencias de la comunicación. Tomando como base la teoría de Usos y Gratificaciones (U\&G), este estudio tiene como objetivo mapear las prácticas de los adolescentes portugueses (13-16) en esta plataforma, explorando las diferencias entre usuarios y creadores de contenido. Para ello, se realizó una encuesta online sobre una muestra no probabilística de 263 usuarios de TikTok. Los resultados muestran que el $44,5 \%$ produce contenido y que tiene un rol más estratégico, enfocado a la autopromoción, expansión y dinamización de su comunidad online.
\end{abstract}


Palabras clave: TikTok, adolescentes (13-16), aplicación móvil, contenidos generados por los usuarios (UGC), media generados por los usuarios (UGM), Usos y Gratificaciones (U\&G).

\section{Introdução}

O TikTok é uma aplicação de criação e partilha de vídeos curtos, lançado em setembro de 2016 e que, desde então, não tem parado de crescer, tendo-se tornado num verdadeiro fenómeno de popularidade à escala global. No entanto, e provavelmente devido à sua novidade, ainda não é vasta a literatura que pode ser encontrada sobre a sua especificidade (Klug, 2020). Consequência da tendência crescente de publicação de conteúdos por parte dos utilizadores, nomeadamente de vídeos de curta duração, do aumento generalizado da difusão da internet de alta velocidade, e da pandemia de Covid-19, que desde março de 2020 obrigou grande parte do mundo a permanecer em casa, aplicações como o TikTok ganharam espaço e importância nos hábitos de socialização humanos, que as Ciências Sociais não podem ignorar.

Desde o surgimento dos chamados media sociais, várias abordagens teóricas tentaram explicar por que razão as pessoas criam, compartilham ou se envolvem com fotos e vídeos nessas plataformas. Este estudo pretende compreender como é que os jovens portugueses (entre os 13 e os 16 anos) estão a utilizar esta aplicação específica e investigar as suas motivações, utilizando como base a teoria dos Usos e Gratificações (U\&G).

De acordo com Feng, Chen e Wu (2019, p. 1), aplicações de vídeos curtos podem ser descritas como "um novo tipo de software social com fácil gravação, reprodução, edição e compartilhamento com base em terminais móveis inteligentes", cuja complexidade dos processos operacionais tem vindo a ser permanentemente simplificada, e algumas funções práticas e instrumentais foram adicionadas para que os utilizadores possam exercer plenamente sua criatividade (Wang, 2020). Esta facilidade de criação é uma das razões que permitiu a sua rápida produção e disseminação (Yang et al., 2020).

A sigla UGC, que significa conteúdo gerado pelos utilizadores, é comumente usada para se referir a uma forma integrada de comunicação que permite que os seus utilizadores criem e compartilhem conteúdo e estabeleçam sua própria rede (Omar \& Dequan, 2020). Essa forma popular de expressão, juntamente com o número crescente de utilizadores, a maior velocidade das conexões de internet, a ascensão da tecnologia móvel, e o valor cada vez maior dos vídeos curtos, deu origem à economia das "celebridades da internet” (Tang, 2019), ou "economia da visibilidade" (Banet-Weiser, 2018). Patel (2020) também reforça essa ideia, mostrando como o TikTok permite que os jovens se estabeleçam como celebridades globais. Efetivamente, o TikTok liberta os jovens para o utilizarem sem aderir aos estilos visuais, narrativas e culturas do passado, sendo uma das suas características fundamentais a sugestão de novas estéticas para os meios 
de entretenimento juvenil (Bresnick, 2019), com van Dijck (2013) a lembrar como os utilizadores de plataformas online adaptaram suas estratégias ao longo dos anos, à medida que se tornaram mais experientes nessas novas "tecnologias de si”.

São inúmeros os estudos que investigaram sobre como os jovens selecionam artefactos de media criados por si para gerir as representações de si mesmos nos media sociais, mas poucos olharam para as práticas, estratégias e motivações.

O objetivo deste estudo é examinar como os adolescentes estão a usar o TikTok e porquê.

\section{Fundamentação teórica}

\subsection{Teoria dos Usos \& Gratificações}

Como McQuail (2009) mencionou, o público não é um recetor passivo. Os estudos da teoria U\&G tratam o público como indivíduos com necessidades específicas e vêm as suas atividades de envolvimento com os diferentes meios de comunicação baseados em motivos de procura específicos, satisfazendo assim suas necessidades, que podem ser agrupadas em quatro grandes categorias: informação, sociabilização, entretenimento e escape (Xu et al., 2019). Como Elliott e Rosenberg (1987, citados em Shao, 2009) escreveram, "sempre que uma nova tecnologia entra no patamar de comunicação de massas, as motivações das pessoas para usar essa tecnologia foram examinadas sob essa perspetiva". A aplicação da teoria U\&G na compreensão do uso do TikTok como um UGC emergente é, por isso, especialmente oportuna (Omar \& Dequan, 2020), uma vez que as plataformas digitais não apenas surgiram como o novo poder moldando comportamentos e atividades online, mas também trouxeram a rutura digital para toda a sociedade (Zhang, 2020). Embora existam muitas críticas a esta teoria, a mesma tem-se mostrado particularmente relevante na análise de meios onde os utilizadores desempenham um papel ativo, como o UGC (Shao, 2009). Apesar de escassos, existem alguns estudos que aplicam a abordagem U\&G ao TikTok. Estes estudos mostram que os utilizadores usam a plataforma para satisfazer necessidades que vão além das categorias mais tradicionais presentes na teoria dos U\&G. É certo que os utilizadores do TikTok buscam informação (como atesta o sucesso dos vídeos DIY), socialização e pertença social (como pode ser observado na replicação de trends, memes e desafios), entretenimento e escape (e por isso a sua utilização aumentou consideravelmente durante o período de confinamento obrigatório devido à pandemia Covid-19, entre março e maio de 2020) (Azerion, 2020). No entanto, os utilizadores buscam mais do que estas dimensões, e obtêm gratificações adicionais.

Chung e Wei (2020) investigaram as razões que levam ao uso continuado da plataforma, concluindo que a satisfação tem um impacto direto nessa prática. Esta satisfação é obtida através da qualidade percebida da aplicação, do serviço 
prestado e do conteúdo. O formato curto dos vídeos e o interface baseado no acto swipe proporcionam satisfação constante, e conferem um caráter particularmente viciante à plataforma (Wang, Gu \& Wang, 2021). Shao e Lee (2020) procuraram estudar as motivações para o uso e sua correspondência com a satisfação e concluíram que a procura de informação e comunicação foram as principais motivações que levaram diretamente à satisfação, enquanto a autoexpressão foi uma motivação importante, mas não impactou diretamente a satisfação. Considerando a estrutura de Shao (2009), a autoexpressão implica contributos, o que émenos comum do que consumir e participar. Segundo Bossen e Kottasz (2020), independentemente da idade, as necessidades de consumo passivo, como vigilância, relaxamento, busca de informação e entretenimento, são, de longe, as gratificações mais importantes procuradas quando se trata do uso de SNS (Social Networking Sites). Já Omar e Dequan (2020) concluíram que escapismo e a interação social são os principais usos dados ao TikTok, e que a autoexpressão é a gratificação mais importante obtida por aqueles que produzem ativamente conteúdo original. Mais recentemente, Wang, Gu e Wang (2021) concluíram que o TikTok satisfaz necessidades cognitivas e emocionais, e destacam a integração social e o alívio da pressão. Scherr e Wang (2021), com um estudo mais abrangente, redefinem os principais usos e gratificações do TikTok em quatro novas categorias: autoexpressão socialmente recompensadora, estar a par de tendências, escapismo viciante, e novidade. Os autores usaram ainda fatores pessoais (como a idade e o género) e contextuais (como a hora de utilização) para encontrar dois perfis: os utilizadores predominantemente diurnos são motivados pela integração social (estar a par de tendências), ao passo que os predominantemente noturnos buscam novidades constantes.

Tendo em conta estes resultados diversificados, o nosso estudo pretende explorar as práticas dos adolescentes portugueses TikTokers e as gratificações que obtêm.

\subsection{Media gerados pelos utilizadores (UGM)}

No final de 2006, a revista americana TIME selecionou o consumidor/utilizador como a "pessoa do ano". Grossman (2006, p. n/a), na época o editor de tecnologia, escreveu: "Por tomar as rédeas dos media globais, por fundar e enquadrar a nova democracia digital, por trabalhar de graça e vencer os profissionais no seu próprio jogo, a pessoa do ano da Time em 2006 é você”. Essa decisão baseou-se na crescente importância que a generalidade dos internautas teve na criação de conteúdos que se propagam por todas as plataformas virtuais, todos os dias, fazendo com que quem contribui com conteúdos para web seja quemimpulsiona a era da informação. Castells (2009) afirma que a comunicação é o poder central na sociedade contemporânea e denominou este sistema global de interação em rede de "autocomunicação em massa". Comunicação de massa, uma vez que tem potencial para atingir um público global, e autocomunicação porque a produção 
da mensagem é autogerada, a definição dos recetores potenciais é autodirigida e a recuperação de mensagens específicas ou o conteúdo da World Wide Web e redes eletrónicas é autoselecionado (Fuchs, 2009).

UGM "refere-se aos novos meios cujo conteúdo é disponibilizado publicamente na Internet, reflete uma certa quantidade de esforço criativo e é criado fora das rotinas e práticas profissionais" (Wunsch-Vincent \& Vichery, 2006, citados em Shao, 2009, p. 8). Segundo Omar e Dequan (2020), o uso de UGM envolve duas atividades importantes: a criação do conteúdo e a sua partilha online. Para Shao (2009), a forma como as pessoas utilizam o UGM pode ser resumida nestas 3 ações: consumir, participar e produzir, onde consumir se refere a quem assiste ou lê, mas nunca participa; participar significa interagir com conteúdos ou com outros utilizadores, mas sem criar nenhum conteúdo; e a produzir, que inclui a criação e publicação de imagens, textos, áudios ou vídeos. Quando cruzamos esta ideia com as quatro razões resumidas por McQuail (2003) para o uso dos media: informação, identidade pessoal, integração, e interação social e entretenimento, Shao $(2009$, p. 9) postula que diferentes usos são movidos por diferentes motivações, desta forma: "as pessoas consomem o conteúdo para informação e entretenimento; participam pela interação social e desenvolvimento comunitário; e produzem conteúdos para autoexpressão e autoatualização".

O surgimento do UGM, no qual o TikTok é a nova estrela em ascensão, reformulou o mundo do compartilhamento de vídeo, onde o conteúdo é agora criado por milhões de utilizadores em vez de um pequeno e controlado número de produtores (Omar \& Dequan, 2020). Como Shao (2009) afirmou, os UGM estavam a mudar o mundo do entretenimento, da comunicação e da informação, especialmente devido à sua natureza autossustentável e a um público cada vez maior. Entre eles, as aplicações de vídeos curtos são muito populares pela facilidade de gravação, edição simples, compartilhamento conveniente e baixos requisitos para habilidades profissionais (Feng et al., 2019; Wang, 2020). Em comparação com as aplicações de vídeo tradicionais, os vídeos destas novas aplicações tendem a ser mais curtos, preenchendo melhor o tempo fragmentado do utilizador (Feng et al., 2019). Aliás, o tempo fragmentado do vídeo é perfeito para os tempos acelerados que vivemos, pois pode ser visto quer no lazer quer no trabalho, o que também é um fator decisivo para facilitar a sua aceitação e divulgação (Yang et al., 2020). Esta popularidade da criação e partilha de vídeos, de vídeos curtos em loop e live-steaming, levou Bresnick (2019) a apelidá-los de "jardim de recreio virtual" e Klug (2020) a afirmar que o TikTok é mais lúdico que social, pois parece mais um jardim de recreio audiovisual experimental do que uma rede social. Isso é especialmente relevante porque o sucesso destes vídeos curtos depende de quantas pessoas os vêm e reagem, o que se reflete no número de "visualizações", "gostos", "partilhas" e "comentários". 


\subsection{TikTok}

O TikTok, conhecido como Douyin na China, é uma aplicação de criação e partilha de vídeos curtos, lançada em setembro de 2016 e detida pela empresa chinesa ByteDance. Em agosto de 2018, fundiu-se com a music.ly, uma aplicação parecida e, desde então, tornou-se num verdadeiro fenómeno global, com o número de utilizadores a crescer sem parar.

Disponível em 154 países e 75 idiomas diferentes, foi descarregado mais de 2,6 biliões de vezes em todo o mundo e tem cerca de 1 bilião de utilizadores ativos por mês, que passam em média 80 minutos por dia na aplicação. O TikTok é a nova obsessão dos jovens, uma fuga da realidade que lhes dá um "palco" para se expressarem de forma inovadora e criativa. A iconografia juvenil, os rituais, os espaços e estilos de vida, podem ser vistos nas suas tendências, danças e desafios. Os vídeos são normalmente filmados em smartphones, em locais "mundanos" como o quarto (Kennedy, 2020) e durante o confinamento global devido à Covid-19, o TikTok viu um aumento extraordinário de utilizadores e visibilidade cultural (Monteiro, 2020), bem como alguns adolescentes tornarem-se celebridades mundiais.

Os utilizadores do TikTok têm, na sua maioria, menos de 20 anos, com crianças entre 4, e 15 anos a passar tanto tempo no TikTok como a assistir a vídeos no YouTube (Kennedy, 2020). No seu estudo, Bossen e Kottasz (2020) confirmaram que as meninas pré-adolescentes eram as maiores utilizadoras do TikTok, quer consumindo, quer participando ou contribuindo, sendo o consumo passivo o comportamento mais usual em grupos de pré e adolescentes, especialmente relacionado com entretenimento e diversão. Para Patel (2020), os jovens, mais que explorar, estão a descobrir o seu potencial através do TikTok, pois podem mostrar o seu talento e encontrar um público sem barreiras de fronteiras nacionais ou limitação de interferências físicas e locais.

$\mathrm{Xu}$, Yan e Zhang (2019) afirmam que o sucesso do TikTok se baseia no que eles chamaram de "promoção de estrelas", usando pessoas influentes e conhecidas. Na verdade, a popularidade de curto prazo de novos produtos, na era da internet móvel, está amplamente relacionada com a recomendação de estrelas e o poder dos fãs (Yang et al., 2020) e são estes líderes de opinião digitais que, devido à sua maior influência, trazem novos utilizadores para a plataforma (Xu et al., 2019). Além disso, o rápido crescimento do TikTok está também relacionado com o seu nível de produção sofisticado, o conteúdo elegante, o poder de criar celebridades globais e as ideias de publicidade interessantes. Outra maneira de olhar para o sucesso do TikTok, de acordo com Yang et al. (2020) é baseado no seu algoritmo, que mostra permanentemente conteúdo de acordo com as preferências e necessidades dos utilizadores. Para Anderson (2020), esse algoritmo torna o TikTok único em relação a outros aplicativos de media sociais que são organizados em torno de quem nos segue e quem seguimos. 
Neste estudo, não pretendemos apenas mapear as práticas dos adolescentes portugueses no TikTok e compreender melhor as suas motivações para o uso da plataforma e as gratificações obtidas, mas também investigar as diferenças entre uma utilização passiva, uma participação ao nível da interação, e a criação de conteúdos (Shao, 2009), procurando compreender o que diferencia estes perfis de utilização.

\section{Metodologia}

\subsection{Questões de Investigação}

Nosso estudo aborda as seguintes questões de pesquisa:

- RQ1. Quais são as práticas dos adolescentes portugueses (13-16 anos) no TikTok? (consumo e produção)

- RQ2. As motivações dos utilizadores e dos criadores de conteúdos para usar a plataforma, e as gratificações obtidas, diferem?

\subsection{Desenho da Investigação e técnica de recolha de dados}

Trata-se de um estudo exploratório, com enfoque no contexto português. $\mathrm{O}$ desenho da pesquisa é simples, com apenas um momento de recolha de dados (Creswell, 2003). Como técnica de recolha de dados, usamos um inquérito por questionário online construído no Google Forms. Essa técnica de recolha de dados é adaptada ao nosso universo, adolescentes entre 13 e 16 anos que utilizam o TikTok, por conhecerem o ambiente digital e terem acesso à internet.

A Tabela 1 apresenta a organização do nosso questionário e a sua fundamentação teórica (de Vaus, 2013).

Tabela 1. Estrutura do questionário

\begin{tabular}{|l|l|l|}
\hline \multicolumn{1}{|c|}{ Partes } & \multicolumn{1}{|c|}{ Questões } & \multicolumn{1}{c|}{ Fundamentação teórica } \\
\hline $\begin{array}{l}\text { Consentimento } \\
\text { Informado } \\
\text { (eliminatório) }\end{array}$ & Consentimento Informado & $\begin{array}{l}\text { Regulamento Geral de Proteção de } \\
\text { Dados-Lei n. 58/2019 }\end{array}$ \\
\hline $\begin{array}{l}\text { Parte 1-Dados } \\
\text { Sociodemográficos }\end{array}$ & $\begin{array}{l}\text { Q1: Idade (eliminatória, apenas entre os 13 e 16 } \\
\text { continuam) }\end{array}$ & Bossen \& Kottasz (2020) \\
\cline { 2 - 4 } & Q2: Género & Kennedy (2020) \\
\cline { 2 - 4 } & Q3: Ano escolar & - \\
\cline { 2 - 4 } & Q4: Distrito de residência & - \\
\hline
\end{tabular}




\begin{tabular}{|c|c|c|}
\hline \multirow[t]{16}{*}{$\begin{array}{l}\text { Parte } 2 \text { - Práticas do } \\
\text { uso do TikTok }\end{array}$} & $\begin{array}{l}\text { Q5: Utilização do TikTok (eliminatória, apenas } \\
\text { utilizadores continuam) }\end{array}$ & - \\
\hline & Q6: Tempo de utilização & Klug (2020) \\
\hline & Q7: Frequência de utilização & Klug (2020) \\
\hline & Q8: Tipo de perfil & Anderson (2020) \\
\hline & Q9: Número que seguem & Wang (2020) \\
\hline & Q10: Número de seguidores & Wang (2020) \\
\hline & Q11: Tipos de vídeo preferidos & Bresnick (2019); Klug (2020) \\
\hline & Q12: Uso de mensagens privadas & Anderson (2020) \\
\hline & $\begin{array}{l}\text { Q13: Motivações e gratificações do uso do } \\
\text { TikTok }\end{array}$ & $\begin{array}{l}\text { Shao \& Lee (2020); Chung \& Wei } \\
\text { (2020); Omar \& Dequan (2020) }\end{array}$ \\
\hline & Q14: Perceção dos riscos e oportunidades & Anderson (2020) \\
\hline & Q15: Perceção da utilização excessiva & Yang, Zhao \& Ma (2019) \\
\hline & $\begin{array}{l}\text { Q16: Criação de conteúdos (eliminatória, apenas } \\
\text { os criadores continuam) }\end{array}$ & \\
\hline & Q17: Tipo de conteúdos criados & $\begin{array}{l}\text { Shao (2009); Bresnick (2019); Klug } \\
\text { (2020) }\end{array}$ \\
\hline & Q18: Referências a marcas & Xu, Yan \& Zhang (2019) \\
\hline & Q19: Frequência de criação de conteúdos & $\begin{array}{l}\text { Shao (2009); Bresnick (2019); Klug } \\
\text { (2020) }\end{array}$ \\
\hline & $\begin{array}{l}\text { Q20: Gratificações obtidas com a criação de } \\
\text { conteúdos }\end{array}$ & Bresnick (2019); Klug (2020) \\
\hline
\end{tabular}

Um relatório da Azerion (2020), representante nacional do TikTok, afirma que a plataforma contava com 1,8 milhões de utilizadores portugueses em maio de 2020 e tinha crescido $36 \%$ desde março, integrando 500 mil novos utilizadores em dois meses. $\mathrm{O}$ nosso inquérito esteve disponível online em novembro e dezembro de 2020.

\subsection{Técnica de análise de dados}

Para analisar nossos dados, usamos estatística descritiva e exploramos algumas correlações usando Excel e SPSS (de Vaus, 2013).

\subsection{Amostragem e Amostra}

Para atingir uma amostra de utilizadores do TikTok, começamos por recrutar voluntários na plataforma, utilizando, assim, uma amostra de conveniência (Kalton, 2020). Identificamos alguns alunos do Ensino Superior que são 
influenciadores no TikTok e pedimos-lhes que fizessem publicações sobre o nosso projeto e compartilhassem o link para o nosso inquérito online. Além disso, para atingir uma amostra mais ampla e diversificada, entramos em contato com diversos centros de atividades, como escolas de dança e clubes desportivos, e solicitamos que divulgassem o link para o nosso inquérito online entre seus membros.

Obtivemos um total de 505 respostas à nossa pesquisa online. Porém, eliminamos 192 respostas por não se enquadrarem na faixa etária entre os 13 e os 16 anos (Q1) e 50 respostas que não usavam o TikTok (Q5), ficando com uma amostra de 263 respostas $(\mathrm{n}=263)$. Embora esta amostra tenha uma dimensão relevante tendo em conta a população portuguesa desta faixa etária, e seja bastante específica pois recrutamos utilizadores do TikTok na própria plataforma, reforçamos que este é um estudo exploratório com uma amostra de conveniência e, portanto, não tem pretensão de ser representativo dos adolescentes portugueses em geral. É um primeiro estudo sobre os adolescentes portugueses que usam o TikTok, no qual tivemos o cuidado de procurar a participação de jovens residentes em diferentes zonas do país, e com estatuto socioeconómico diverso. Além disto, a caracterização da nossa amostra, na qual predominam utilizadoras do género feminino (84\%) e residentes em zonas urbanas (76\%), é consistente com os dados apresentados pela Azerion (2020) para o país.

\section{Resultados e discussão}

\subsection{Práticas de uso do TikTok dos adolescentes portugueses}

A Azerion (2020) afirma que, em média, o aplicativo TikTok é aberto em torno de 7 vezes ao dia, e os utilizadores dedicam-lhe um total de 50 minutos diários. $\mathrm{O}$ nosso inquérito fornece mais informações sobre o que os utilizadores portugueses fazem nesta plataforma (RQ1). Tal como observado por Shao (2009), constatamos que há mais utilizadores passivos, ou que apenas reagem aos conteúdos da plataforma - 55,5\% - do que utilizadores que são produtores de conteúdos ativos, estes perfazem $45,5 \%$. Ainda assim, a quantidade de utilizadores que são produtores de conteúdos na nossa amostra é elevada.

Em relação ao tempo de uso (Q6), apesar do rápido crescimento da plataforma em Portugal desde o início da pandemia Covid-19 (Azerion, 2020), observamos que 40,1\% dos adolescentes inquiridos já utilizam o TikTok há mais de 2 anos. Observamos também uma maior quantidade de produtores de conteúdos entre quem utiliza o TikTok há mais tempo, pois $61,8 \%$ dos produtores de conteúdos usam o TikTok há mais de 2 anos. 
Figura 1. Há quanto tempo os inquiridos usam o TikTok, comparando utilizadores e produtores de conteúdos (em percentagem)



Reltivamente à frequência de uso (Q7), a maioria dos utilizadores abre o aplicativo com frequência, o que também é consistente com o relatório da Azerion (2020). É mais frequente uma utilização mais intensa entre os produtores de conteúdos, $38,5 \%$ deles abre o aplicativo mais de 10 vezes por dia.

Figura 2. Frequência com que os inquiridos abrem o aplicativo TikTok, comparando utilizadores e produtores de conteúdos (em percentagem)

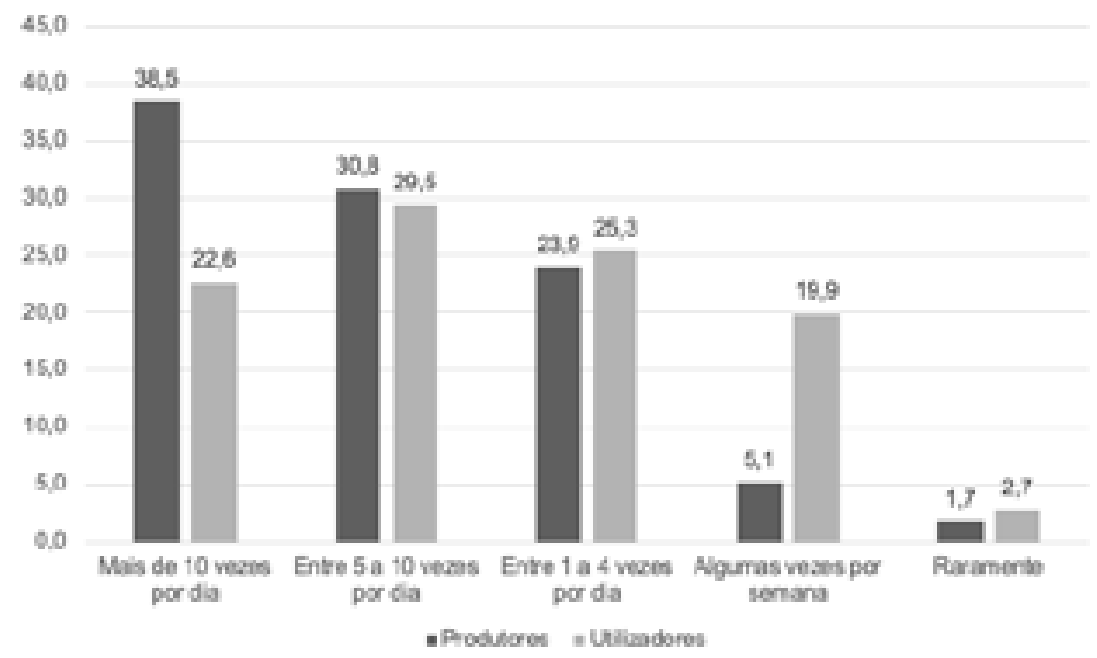


No que diz respeito à segurança e privacidade (Q8), é notória a diferença entre os produtores de conteúdos, entre os quais $76,1 \%$ têm os seus perfis públicos, e os que não produzem conteúdos, entre os quais apenas $52,1 \%$ têm os seus perfis públicos.

A mesma tendência é observável no uso das mensagens privadas da plataforma (Q12), realizado por 59\% dos produtores de conteúdos e apenas por $37 \%$ dos utilizadores. A atuação dos utilizadores é consistente com o estudo de Shao e Lee (2020), que demonstra que o TikTok é mais usado para entretenimento do que para comunicação. A idade pode também ser um fator que conduz a um maior resguardo da privacidade, pois a mediação parental das tecnologias digitais enfatiza muito a segurança, sobretudo no caso dos mais jovens, e desaconselha perfis públicos bem como a comunicação com estranhos (Livingstone, Ólafsson, Helsper, Lupiáñez-VIllanueva, Veltri \& Folkvord, 2017). O comportamento contrário dos produtores de conteúdos uma estratégia ativa por parte dos produtores de conteúdos, que procuram ativamente expandir a sua rede e manter uma comunidade de seguidores ativa (Klug, 2020).

Figura 3. Tipo de perfis dos inquiridos e utilização das mensagens privadas no TikTok, comparando utilizadores e produtores de conteúdos (em percentagem)

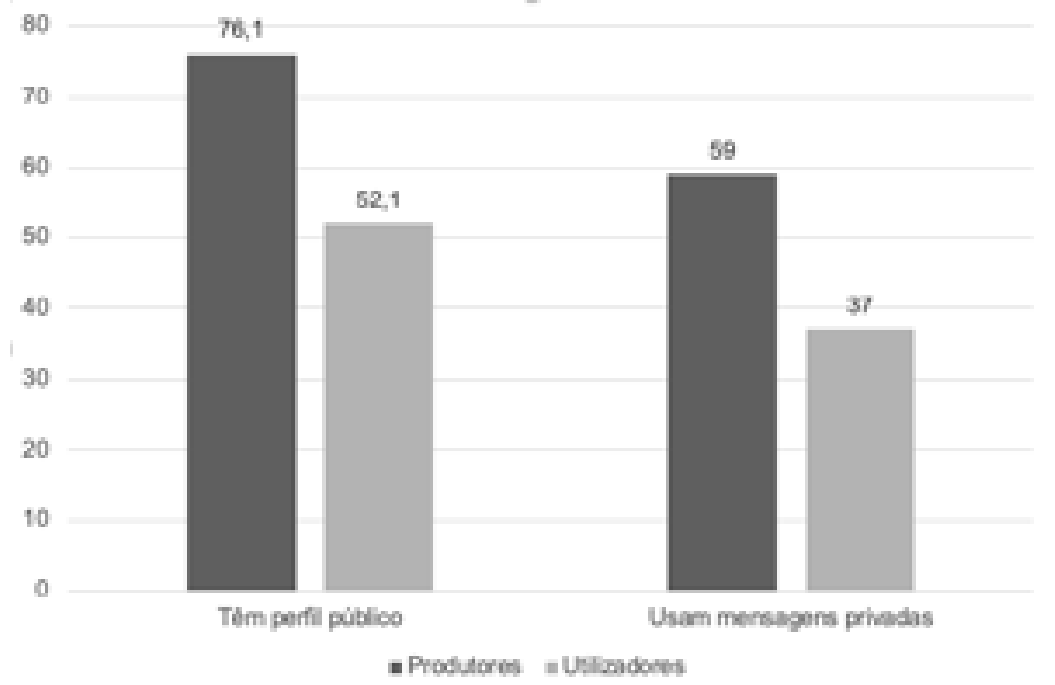

No que diz respeito ao número de perfis que cada utilizador segue (Q9) e ao número de seguidores (Q10), utilizamos como referência os 1000 seguidores, uma vez que Xu et al. (2019) classificam os utilizadores que têm entre $1000 \mathrm{e}$ 10000 seguidores como nano-influenciadores. Observamos que os utilizadores têm redes mais pequenas do que os produtores de conteúdos, e que tendem a seguir mais perfis do que o número de seguidores que apresentam. No caso dos 
produtores de conteúdos, $33,4 \%$ atingem o estatuto de nano-influenciadores e, ao contrário, o seu número de seguidores tende a ser superior ao número de perfis que seguem.

Figura 4. Inquiridos que seguem mais de 1000 perfis e que têm mais de 1000 seguidores no TikTok, comparando utilizadores e produtores de conteúdos (em percentagem)

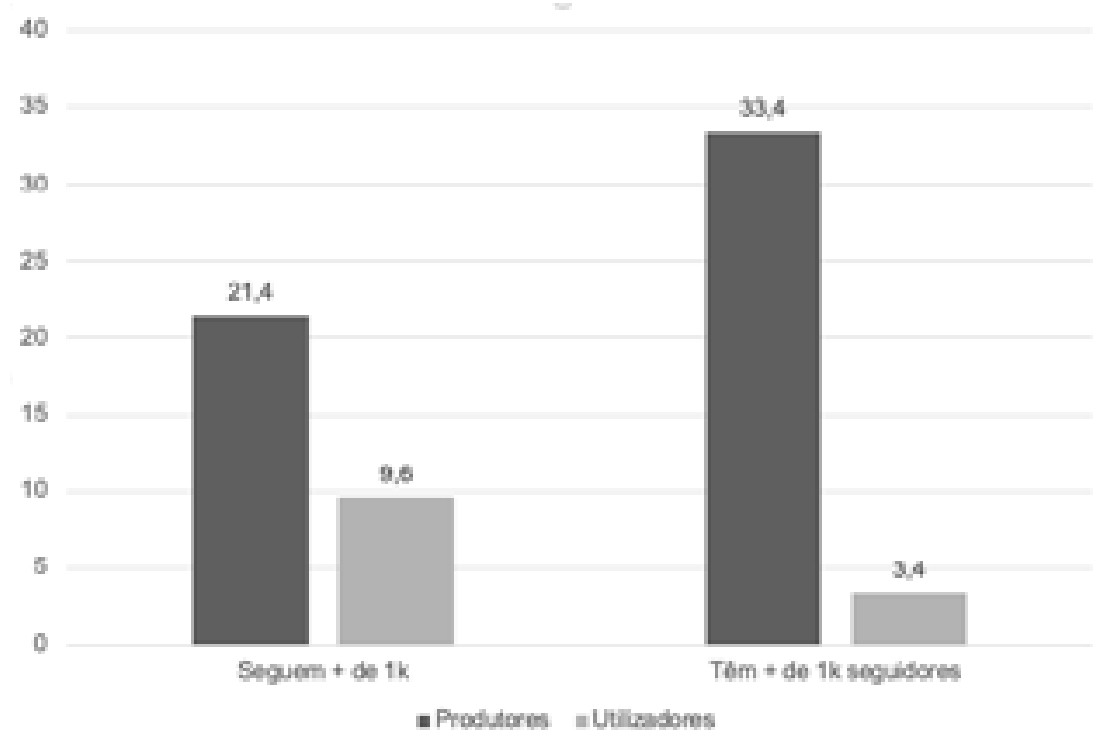

Perguntamos aos nossos entrevistados sobre suas preferências em relação ao conteúdo do TikTok (Q11). Os resultados destacam os vídeos de coreografias, os vídeos humorísticos e as canções, em linha com pesquisas anteriores (Bresnick, 2019). No entanto observam-se ligeiras diferenças entre os utilizadores da plataforma e os produtores de conteúdos. Os utilizadores preferem vídeos humorísticos $(79,5 \%)$, vídeos de coreografias $(76,7 \%)$ e POV's (representações dramáticas curtas intituladas "Pontos de Vista") (70,5\%). Destacam-se também os desafios $(69,2 \%)$. Os produtores de conteúdos preferem vídeos de coreografias $(88,9 \%)$, canções $(81,2 \%)$ e vídeos humorísticos $(81,2 \%)$. Destacam também outros formatos característicos do TikTok (além das coreografias), como os POV's $(78,6 \%)$, as transições $(68,4 \%)$ e as rotinas $(65 \%)$, e ainda conteúdos mais ligados a marcas, como os tutoriais de moda $(67,5 \%)$. 
Figura 5. Conteúdos do TikTok de que os inquiridos gostam, comparando utilizadores e produtores de conteúdos (em percentagem)

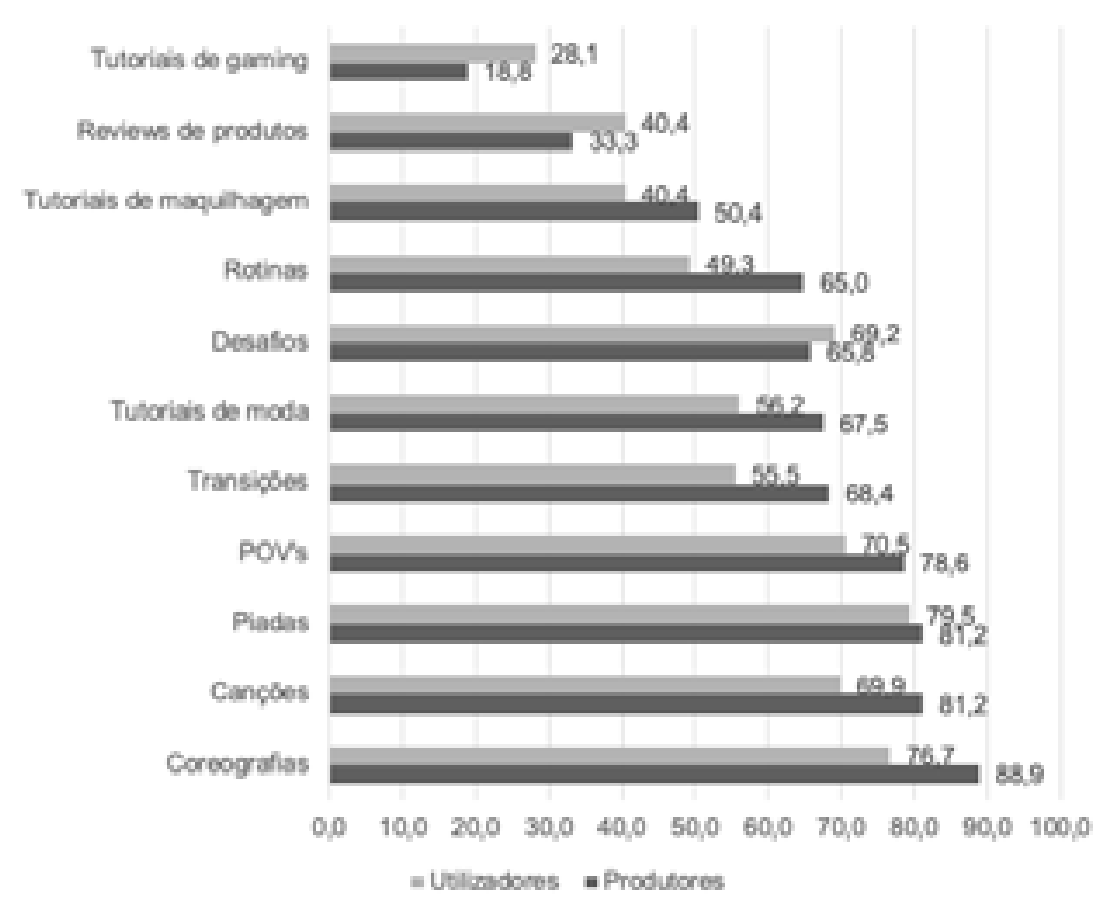

Da nossa amostra, são 44,5\% os que se declaram criadores de conteúdos (Q16). Sobre o tipo de conteúdo que os nossos respondentes criam (Q17), observamos uma correspondência entre as tipologias produzidas e as preferidas, com destaque para os vídeos de coreografias (produzidos por $63,2 \%$ dos produtores de conteúdos, e preferidos por $82,1 \%$ da nossa amostra), as POV's (produzidos por $42,7 \%$ dos produtores de conteúdos, e preferidos por $74,9 \%$ da nossa amostra) e as transições (produzidos por 33,3\% dos produtores de conteúdos, e preferidos por $80,2 \%$ da nossa amostra), todos estes formatos muito característicos desta plataforma. As tipologias que costumam ser produzidas por influenciadores, como tutoriais e reviews, não têm grande expressão entre os produtores de conteúdos da nossa amostra, que se mostram alinhados com as preferências dos utilizadores do TikTok (Klug, 2020). Embora muitos TikTokers busquem mais seguidores e tenham a ambição de chamar a atenção das marcas para que possam monetizar essa atividade (Patel, 2020), são apenas 9,4\% os que incluem referências intencionais a marcas nas suas publicações. 
Figura 6. Conteúdos do TikTok que os produtores de conteúdos criam, comparativamente aos preferidos de todos os inquiridos (em percentagem)

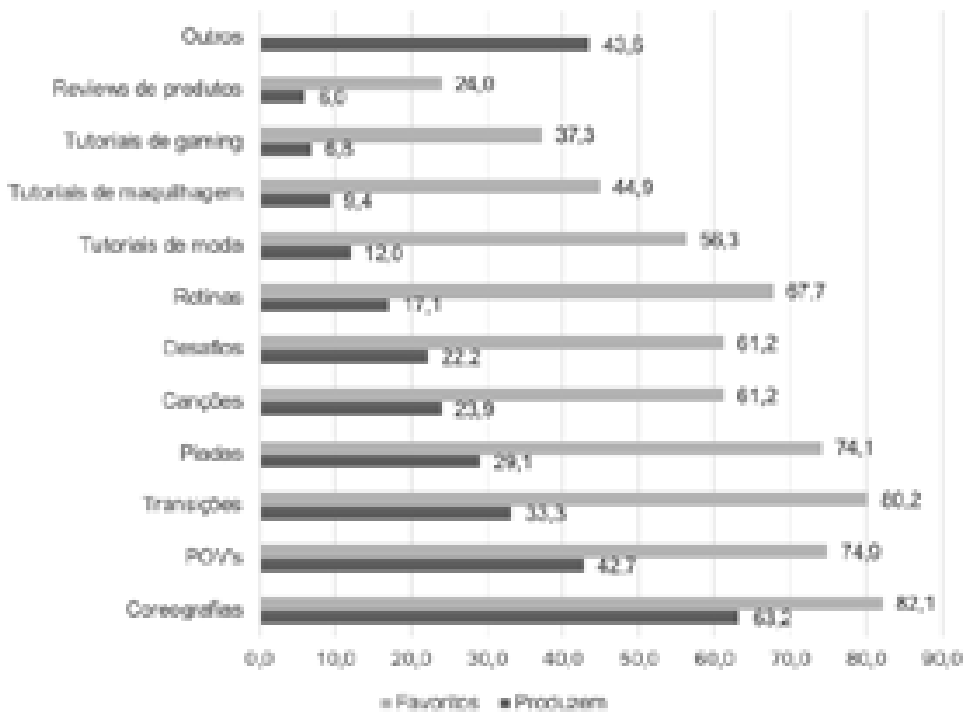

Embora alguns dos produtores de conteúdos na nossa amostra aspirem ser “TikTokers” e demonstrem preocupação com a gestão da sua comunidade de seguidores online, não produzem conteúdos com muita frequência (Q19) (Shao, 2009). Apenas 14,5\% publica pelo menos diariamente, e são $20,5 \%$ os que têm um ritmo semanal de publicação.

Figura 7. Frequência de criação de conteúdo no TikTok (em percentagem)

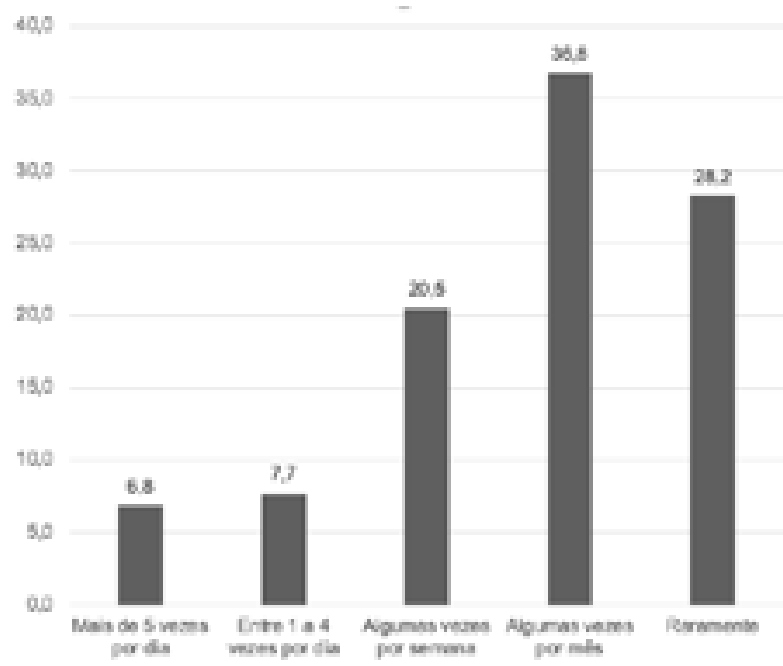




\subsection{Motivações e gratificações do TikTok, entre os jovens portugueses}

Relativamente às motivações da nossa amostra para usar o TikTok (RQ2), os tipos de vídeos preferidos (Q11) já apontam pistas. Os vídeos de coreografias, piadas e canções são conteúdos artísticos e humorísticos, que respondem a uma necessidade ou desejo de entretenimento e autoexpressão. Este é um cenário diferente do descrito por Shao e Lee (2020) na China, em que a maioria dos utilizadores do TikTok procurava principalmente informação e comunicação e não criava conteúdo original; e mais consistente com a descrição de Omar e Dequan (2020), que identificam o escapismo e a interação social como usos importantes da plataforma. Ao perguntar sobre o nível de concordância dos nossos participantes com várias afirmações sobre as motivações para usar o TikTok, usando uma escala entre 1 - "discordo totalmente" e 5 - "concordo totalmente" (Q13), destaca-se o entretenimento, pois 97,9\% dos utilizadores e 97,4\% dos produtores de conteúdos concordam com a afirmação "Eu uso o TikTok para me distrair”. Os produtores de conteúdos mostram-se ligeiramente mais motivados pela busca de informação noticiosa (94,9\%) e sobre interesses pessoais (98,3\%), bem como pela aprendizagem (94,9\%). A autoexpressão é também uma motivação mais forte entre os produtores de conteúdos, tal como afirmam Omar e Dequan (2020), tendo 95,7\% da nossa amostra concordado com a afirmação "Eu uso o TikTok para expressar a minha criatividade", 90,6\% com "Eu uso o TikTok para expressar as minhas opiniões", e 81,2\% com "Eu uso o TikTok para expressar a minha identidade”.

Figura 8. Concordância com expressões sobre motivações para usar o TikTok, comparando utilizadores e produtores de conteúdos (em percentagem)

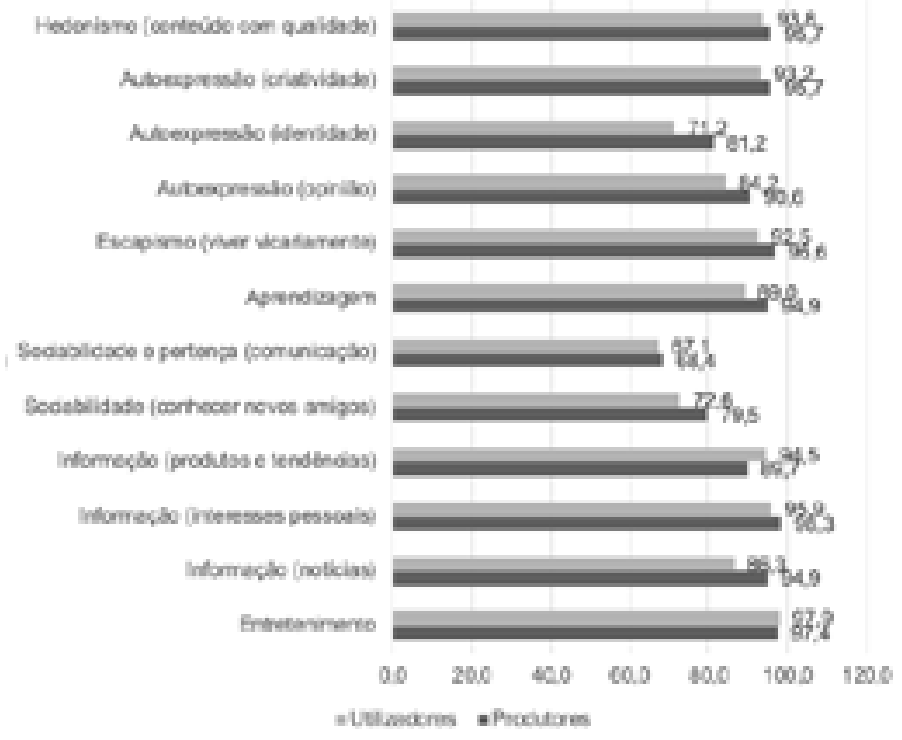


Relativamente às gratificações obtidas, destaca-se a diversão, tendo 98,6\% dos utilizadores e 97,4\% dos produtores de conteúdos concordado com a afirmação "O TikTok diverte-me", e 94,5\% e 96,6\%, respetivamente, com "O TikTok faz-me sentir bem”. Destaca-se também o hedonismo, tendo 95,2\% dos utilizadores e 94,9\% dos produtores de conteúdo afirmado que disfrutam e apreciam a estética característica da plataforma, tal como nos estudos de Chung e Wei (2020). Uma percentagem ligeiramente mais elevada de produtores de conteúdos reconhece gratificações como o desenvolvimento de competências digitais $(93,2 \%)$, o escapismo (89,7\%) e a criação de conexões com outras pessoas $(80,3 \%)$. A pertença a uma comunidade é a gratificação menos reconhecida pela nossa amostra, embora figure nos estudos de Shao e Lee (2020).

Figura 9. Concordância com expressões sobre gratificações obtidas a partir do TikTok, comparando utilizadores e produtores de conteúdos (em percentagem)

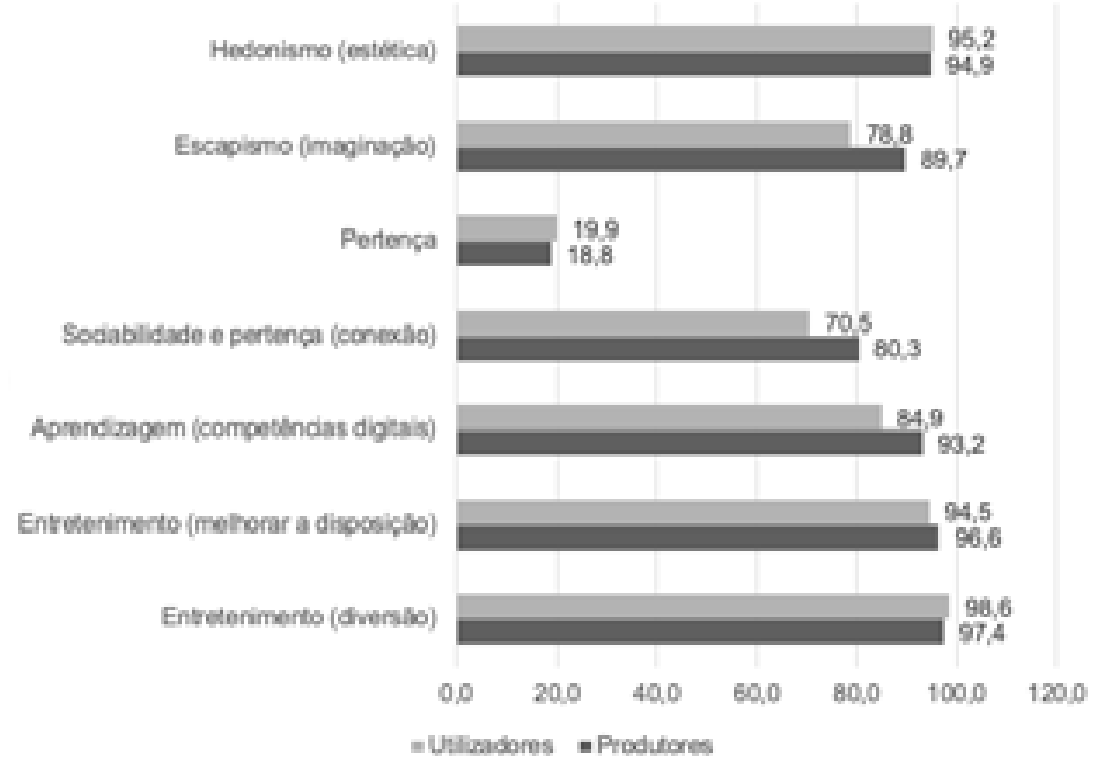

No âmbito da teoria dos U\&G, a conceito de gratificação é geralmente benéfico para o utilizador de um determinado meio. No entanto, questionamos os nossos inquiridos sobre consequências negativas resultantes do uso utilização do TikTok (Q14 e Q15).

A maior preocupação dos nossos respondentes é a exposição a notícias faltas no TikTok, para 96,6\% dos produtores de conteúdos e para 93,8\% dos utilizadores. A exposição ao bullying é o aspecto negativo que reúne menos concordância, mas ainda assim é reportado por $41,4 \%$ dos utilizadores da plataforma, e por $39,3 \%$ 
dos produtores de conteúdos. A maior diferença entre estes dois grupos surge nos temas da utilização excessiva e do vício, sendo que $76,1 \%$ dos produtores de conteúdos consideram que usam o TikTok excessivamente e 71,8\% que são viciados, face a apenas $54,1 \%$ e $52,7 \%$ dos utilizadores, o que também é observado na frequência de uso reportada $(\mathrm{Q} 7)$.

Figura 10. Concordância com expressões sobre consequências negativas da utilização do TikTok, comparando utilizadores e produtores de conteúdos (em percentagem)

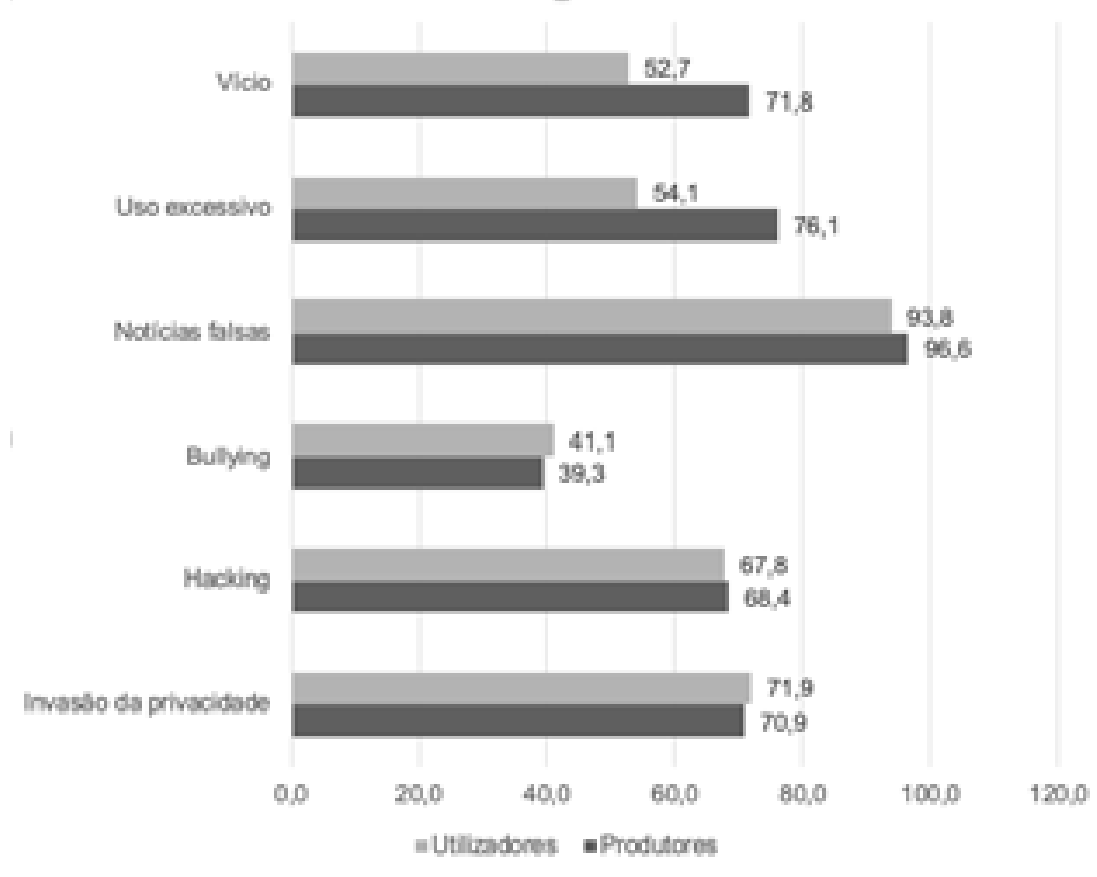

Por fim, investigamos as motivações específicas para a criação de conteúdo, e se os seus autores retiram gratificações adicionais dessa atividade (Q16).

Já vimos que perfis públicos (Q8) e o uso de mensagens privadas na plataforma (Q12) são mais frequentes entre os produtores de conteúdos, o que é indicativo da importância que dão à expansão da sua rede e à dinamização da sua comunidade de seguidores, bem como constatamos que têm redes mais alargadas (Q9 e Q10). No entanto, vimos também que a comunicação (Q11) apenas é motivação para o uso do TikTok para 68,4\% dos produtores de conteúdos. Embora 74,4\% incluam perguntas nas suas publicações para motivarem os seus seguidores a interagirem com eles, e 86,3\% respondam sempre aos seus seguidores, podemos inferir que a comunicação em si não é o que buscam, sendo esta uma estratégia para atingir o objetivo a que $68,4 \%$ afirmam aspirar, serem TikTokers profissionais (Bossen e Kottasz, 2020; Omar \& Dequan, 2020). Em linha com Klug (2020), que destaca 
a autoexpressão e a autopromoção como os principais usos do TikTok, os nossos respondentes dão grande importância a agradarem aos seus seguidores $(75,2 \%)$ e a obterem "gostos" nas suas publicações $(72,6 \%)$. Com essas ações, não buscam a interação social ou o sentimento de pertença em si, mas sim aumentar o alcance dos seus perfis e reforçar o grupo de fãs, eventualmente atraindo a atenção de marcas, e aspirando a profissionalizar a sua atividade de produtores de conteúdos.

Figura 10. Concordância com expressões sobre motivações para a criação de conteúdos no TikTok e gratificações obtidas dessa atividade (em percentagem)

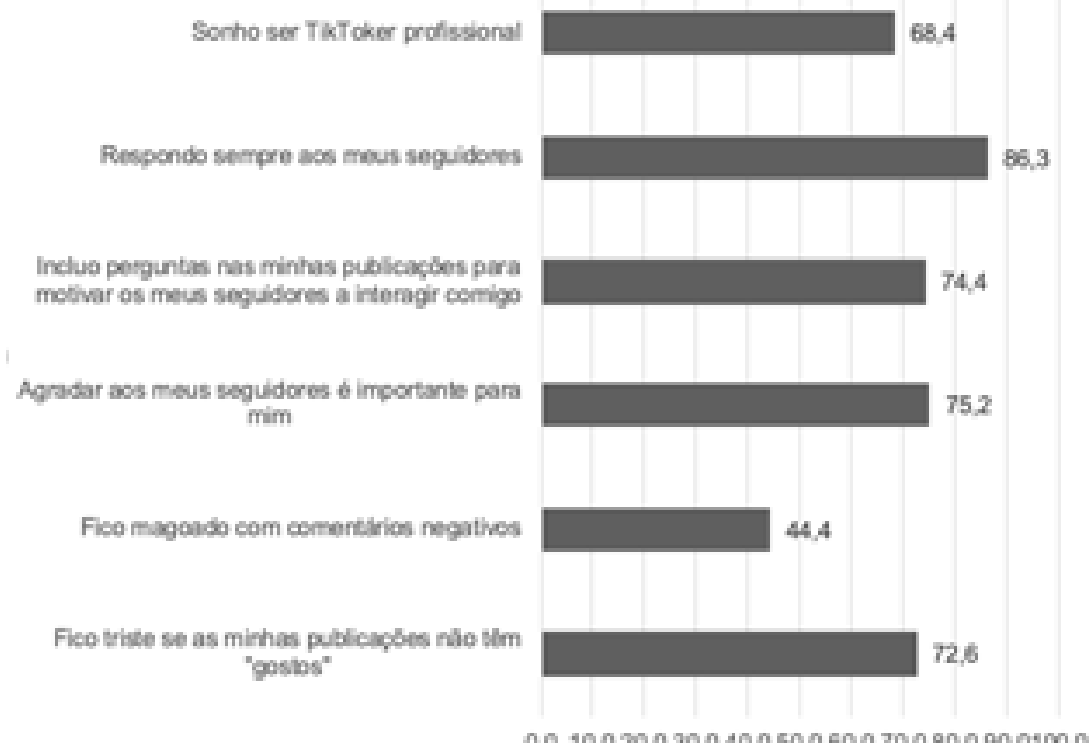

\section{Conclusão}

Este estudo exploratório traça um panorama das práticas dos adolescentes portugueses (13-16 anos) no TikTok. De forma consistente com pesquisas anteriores, a maioria dos utilizadores do TikTok tende a ser passiva ou participante, em vez de contribuir ativamente (Shao, 2009), apenas 44,5\% da nossa amostra é produtora de conteúdos.

A teoria dos U\&G constituiu um enquadramento útil para explorar as motivações de uso desta plataforma, bem como as gratificações obtidas. Resultados anteriores apontaram para direções diferentes, e os nossos sugerem que ser um utilizador ou um produtor de conteúdos (Shao, 2009) faz a diferença. 
Por um lado, encontramos mais semelhanças do que diferenças entre os vários perfis e graus de atividade dos utilizadores do TikTok. Por exemplo, estão alinhados relativamente aos tipos de vídeos que preferem, e aos que produzem. Destaca-se o surgimento de novos tipos de conteúdos de vídeo curto, como coreografias, POVs, transições e rotinas diárias, que podem ser considerados nativos do TikTok, e que reúnem a preferência de grande parte da nossa amostra.

No entanto, é pertinente um estudo mais aprofundado dos usos e das gratificações obtidas por diferentes perfis de utilizador, seguindo as descobertas de Scherr e Wang (2021), que apontam para uma diferenciação de necessidades que utilizadores do TikTok e produtores de conteúdos no TikTok procuram satisfazer, e também para uma diferenciação das gratificações obtidas.

Os utilizadores portugueses procuram e obtêm, principalmente, entretenimento - não informação ou interação social como Shao e Lee (2020) encontraram. Já os produtores de conteúdos são motivados pela autoexpressão e gratificados com a aprendizagem, como Bossen e Kottasz (2020) também descobriram. A interação e a integração sociais parecem ser gratificações obtidas, embora não inicialmente procuradas. É observável uma utilização mais "estratégica" por parte dos produtores de conteúdos quanto às suas contribuições e também às suas configurações de privacidade, mais focada na construção de uma imagem social e de um público (Bossen \& Kottasz, 2020), fazendo a transição da autoexpressão para a autopromoção como principal motivação para usar o TikTok (Klug, 2020). Consistentemente com os argumentos de Patel (2020) e Klug (2020), os adolescentes usam o TikTok para se autopromover, e a interação social é um meio de promover e nutrir a gratificação que eles realmente buscam, reconhecimento social e admiração, e até celebridade, atingindo, portanto, uma autoexpressão socialmente recompensadora, como teorizado por Scherr e Wang (2021).

Uma particularidade do panorama português do TikTok que merece um estudo mais aprofundado é a maior diversidade de perfis do que a "menina adolescente branca e saudável" (Kennedy, 2020), já que os 10 perfis principais na plataforma incluem cinco meninos, quatro meninas e um perfil humorístico inteiramente dedicado a memes (Azerion, 2020).

Scherr e Wang (2021) abordam a natureza viciante do TikTok, e associam-na às necessidades de estar a par das tendências e da procura de novidades. Estas dimensões não foram exploradas no nosso estudo, mas é uma linha de investigação que importa aprofundar.

Por ser exploratório, este estudo aponta vários caminhos de investigação futura. Validar estes resultados com uma amostra mais ampla e probabilística seria importante para melhor traçar o panorama da utilização do TikTok entre os adolescentes portugueses. Além disso, a pesquisa qualitativa pode lançar mais luz sobre as motivações para usar o TikTok e as gratificações obtidas, particularmente pela criação de conteúdo, e também sobre os perfis de TikTokers ativos e sua contribuição para esta cultura digital jovem 
contemporânea emergente (Klug, 2020). Finalmente, considerando as preocupações de van Dijck (2013) sobre a natureza algorítmica e comercial dessas plataformas aparentemente "gratuitas", explorar mais a fundo o papel desempenhado por influenciadores e marcas na TikTok é fundamental, bem como a literacia mediática, e em particular para a publicidade, dos utilizadores desta plataforma.

\section{Referências bibliográficas}

Anderson, K. E. (2017). Getting acquainted with social networks and apps: periscope up! Broadcasting via live streaming. Library Hi Tech News.

Anderson, K. E. (2020). Getting acquainted with social networks and apps: it is time to talk about TikTok. Library Hi Tech News.

Azerion (2020). "Tik Tok em Portugal". Lisbon: Lisbon Digital School, May 20 th 2020. Retrieved from $\underline{\text { https://bit.ly/2XNCZEJ }}$

Banet-Weiser, S. (2018). Empowered: Popular feminism and popular misogyny. Duke University Press.

Bereznak, A. (2019), "Memes are the new pop stars: how TikTok become the future of the music industry", The Ringer, 27th June, available at https://www.theringer.com/ tech/2019/6/27/18760004/tiktok-old-town-road-memes-music-industry (accessed 19 December 2020).

Bossan, C. B., \& Kottasz, R. (2020), "Uses and gratifications sought by pre-adolescent and adolescent TikTok consumers", Young Consumers, Vol. 21, n. 4, p. 463-478.

Bresnick, E. (2019). Intensified Play: Cinematic study of TikTok mobile app. Research Gate, available at: www. researchgate. net/publication/335570557_Intensified_Play_Cinematic_study_of_TikTok_mobile_app (accessed 5 January 2021).

Chaudron, S., Di Gioia, R., \& Gemo, M. (2018). Young children (o-8) and digital technology, a qualitative study across Europe. EUR 29070; doi:10.2760/294383. https://bit.ly/2XwYDNn

Courtois, C., Mechant, P., De Marez, L., \& Verleye, G. (2009). Gratifications and seeding behavior of online adolescents. Journal of Computer-Mediated Communication, 15(1), 109-137.

Chung, Y., \& Wei, X. (2020). Effects of Perceived Quality of TikTok on User Satisfaction and Continuance Intention and the Role of Commercial Viability. The Journal of Korea Contents Association 20(6), 77-89. https://doi.org/10.5392/JKCA.2020.20.06.077

Creswell, J. (2003). Research Design, Qualitative, Quantitative, and Mixed Methods Approaches. Thousand Oaks: Sage Publications.Creswell, J. (2003). Research Design: Qualitative, Quantitative and Mixed Approaches. London: Sage.

Da Silva Monteiro, J. C. (2020). Tiktok como novo suporte midiático para a aprendizagem criativa. Revista Latino-Americana de Estudos Científicos, 05-20.

De Vaus, D. (2013). Surveys in Social Research. London: Routledge.

Feng, Y. L., Chen, C. C., \& Wu, S. M. (2019, November). Evaluation of Charm Factors of Short Video User Experience using FAHP-A Case Study of Tik Tok APP. In IOP Conference Series: Materials Science and Engineering (Vol. 688, n. 5, p. 055068). IOP Publishing.

Fuchs, C. (2009). Some Reflections on Manuel Castells' Book "Communication Power". tripleC: Communication, Capitalism \& Critique. Open Access Journal for a Global Sustainable Information Society, 7(1), 94-108. 
Grossman, L. (2006). Person of the year: You. Time, December,13.

Haigney, S. (2020) TikTok is the perfect medium for the splintered attention spans of lockdown. The Guardian, 16 May. Available at https://www.theguardian.com/commentisfree/2020/may/16/tiktok-perfect-medium-splintered-attention-spans-coronavirus-lockdown (accessed 20 December 2020)

Hauben, M. (1995) 'The Net and netizens: the impact the Net has on people's lives', http:// www.columbia.edu/ rh120/ch106.x01, accessed 23 December 2020.

Hern, A. (2019). Adults don't get it”: Why TikTok is facing greater scrutiny. The Guardian, 5 July. Available at: https://www.theguardian.com/technology/2019/jul/05/why-tiktok-isfacing-greater-scrutiny-video-sharing-app-child-safety (accessed 22 December 2020)

Kalton, G. (2020). Introduction to Survey Sampling. London: Sage.

Katz, E. (1974). Utilization of mass communication by the individual. The uses of mass communications: Current perspectives on gratifications research, 19-32.

Kennedy, M. (2020). 'If the rise of the TikTok dance and e-girl aesthetic has taught us anything, it's that teenage girls rule the internet right now': TikTok celebrity, girls and the Coronavirus crisis. European Journal of Cultural Studies, 23(6), 1069-1076.

Klug, D. (2020). "It took me almost 30 minutes to practice this". Performance and Production Practices in Dance Challenge Videos on TikTok. arXiv preprint arXiv:2008.13040.

Laermer, R., \& Simmons, M. (2008). Punk marketing: junta-te à revolução. Leya.

Leung, L. (2009). User-generated content on the internet: an examination of gratifications, civic engagement and psychological empowerment. New media \& society, 11(8), 1327-1347.

Livingstone, S., Óláfsson, K, Helsper, E., Lupiáñez-Villanueva, F., Veltri, G., \& Folkvord, F. (2017). Maximizing Opportunities and Minimizing Risks for Children Online: The Role of Digital Skills in Emerging Strategies of Parental Mediation. Journal of Communication, 67(1), 82-105. https://doi.org/10.1111/jcom.12277

McQuail, D. (1984). With the benefit of hindsight: Reflections on uses and gratifications research. Critical Studies in Media Communication, 1(2), 177-193.

McQuail, D. (2003). Teoria da Comunicação de Massas. Lisboa: Calouste Gulbenkian.

Musso, P. (2004). A filosofia da rede. Tramas da rede: novas dimensões filosóficas, estéticas e políticas da comunicação. Porto Alegre: Sulina, 17-38.

O'Hara, K., Mitchell, A. S., \& Vorbau, A. (2007). Consuming video on mobile devices. In Proceedings of the SIGCHI conference on Human factors in computing systems (p. 857-866).

Omar, B., \& Wang, D. (2020). Watch, share or create: The influence of personality traits and user motivation on TikTok mobile video usage. International Journal of Interactive Mobile Technologies, 14(4), 121-137.

Paasonen, S. (2018). Affect, data, manipulation and price in social media. Distinktion: Journal of social theory, 19(2), 214-229.

Patel, K. (2020). Tik Tok the New Alternative Media for Youngsters for Online Sharing of Talent: An Analytical Study. Available at SSRN 3600119.

PORDATA (2020). "População Residente: Total e por grandes grupos". Retrieved from https:// bit.ly/2XCrEHG

Ponte, C., Simões, J. A., Batista, S., \& Jorge, A. (2017). Crescendo entre Ecrãs: O uso de meios tecnológicos por crianças (3 a 8 anos). Entidade Reguladora para a Comunicação Social (ERC). Retrieved from https://bit.ly/3.9hwteM

Ponte, C., \& Batista, S. (2019). EU Kids Online Portugal. Usos, competências, riscos e mediações da internet reportados por crianças e jovens (9-17 anos). EU Kids Online e NOVA FCSH. https://bit.ly/2XnKClm 
Scherr, S., \& Wang, K. (2021). Explaining the success of social media with gratifications niches: Motivations behind daytime, nighttime, and active use of TikTok in China. Computers in Human Behavior, 124. https://doi.org/10.1016/j.chb.2021.106893

Shao, G. (2009). Understanding the appeal of user-generated media: a uses and gratification perspective. Internet research, Vol. 19 (1), 7-25.

Shao, J. H., \& Lee, S. K. (2020). The Effect of Chinese Adolescents' Motivation to Use TikTok on Satisfaction and Continued Use Intention. The Journal of the Convergence on Culture Technology 6(2), 107-115. https://doi.org/10.17703/JCCT.2020.6.2.107

Takahashi, M., Fujimoto, M., \& Yamasaki, N. (2003, November). The active lurker: influence of an in-house online community on its outside environment. In Proceedings of the 2003 international ACM SIGGROUP conference on Supporting group work (p. 1-10).

Tang, D. (2019). The New Situation of Marketing in the Self-Media Era-Taking Tik Tok as an Example.

Wang, Y. (2020). Humor and camera view on mobile short-form video apps influence user experience and technology-adoption intent, an example of TikTok (DouYin). Computers in Human Behavior, 110, 106373.

Wang, Y. H., Gu, T. J., \& Wang, S. Y. (2021). Causes and characteristics of short video platform internet community taking the TikTok short video application as an example. IEEE International Conference on Consumer Electronics-Taiwan (ICCE-TW). IEEE Xplore. http://doi.org/10.1109/ICCE-TW46550.2019.8992021

Yang, S., Zhao, Y., \& Ma, Y. (2019, July). Analysis of the Reasons and Development of Short Video Application-Taking Tik Tok as an Example. In Proceedings of the 2019 9th International Conference on Information and Social Science (ICISS 2019), Manila, Philippines (p. 12-14).

Xu, L., Yan, X., \& Zhang, Z. (2019). Research on the causes of the "Tik Tok" app becoming popular and the existing problems. Journal of Advanced Management Science Vol, 7(2). Zhang, Z. (2020). Infrastructuralization of Tik Tok: Transformation, power relationships, and platformization of video entertainment in China. Media, Culture \& Society, 0163443720939452 . 
